\title{
Central orderings for the Newton interpolation formula
}

\author{
J. M. Carnicer • Y. Khiar • J. M. Peña
}

\begin{abstract}
The stability properties of the Newton interpolation formula depend on the order of the nodes and can be measured through a condition number. Increasing and Leja orderings have been previously considered [4], [1]. We analyze central orderings for equidistant nodes on a bounded real interval. A bound for conditioning is given. We demonstrate in particular that this ordering provides a more stable Newton formula than the natural increasing order. We also analyze a central ordering with respect to the evaluation point, which provides low bounds for the conditioning. Numerical examples are included.
\end{abstract}

Keywords Newton interpolation formula $\cdot$ conditioning $\cdot$ central ordering

Mathematics Subject Classification (2000) 65D05 - 65F35 - 41A05 • $41 \mathrm{~A} 10$

\section{Introduction}

The Newton interpolation formula is a common representation of Hermite interpolating polynomials at a sequence of nodes. Whereas the Lagrange formula provides an explicit representation of the solution of the Lagrange interpolation problem in terms of the function values, the Newton formula gives rise to solutions of Hermite problems when the nodes coallesce. Nice properties of the stability of the Lagrange basis were described in [2]. In [1], a conditioning associated to a representation of the interpolating polynomial was introduced and the optimal stability of the Lagrange representation with respect to this conditioning was proved for an arbitrary sequence of nodes. As for Newton representations, it is known that a Leja ordering of the nodes has a better

E-mail: yasmina@unizar.es

Departamento de Matemática Aplicada/IUMA, Universidad de Zaragoza, Spain 
global behaviour with respect to the roundoff error properties than the natural increasing order. However, tight bounds for the conditioning of the Newton representation are difficult to obtain. Since the stability of the Newton representation depends on the ordering of the nodes, it can be much more unstable than the Lagrange representation. This motivates to explore simple alternative criteria for the ordering of the nodes providing good stability results. This paper presents central orderings and analyzes the corresponding conditionings of the Newton representations.

In Section 2, we introduce basic concepts related to the Newton formula. We recall how the ordering of the nodes for the Newton formula is of utmost importance and describe Leja orderings. We recall that for the polynomial Newton formula with $n+1$ equidistant nodes in increasing order, the best uniform bound of its conditioning is $3^{n}$ and it is attained at the last node (see Corollary 12 of [1]). In Section 3, we present the central order with respect to a center for any set of nodes. We analyze the relevant case of equidistant nodes. In Theorem 3.1, we provide an upper bound for its conditioning. Numerical examples comparing this order with increasing order and Leja order are included.

More stability is gained using central ordering around the evaluation point, which is analyzed in Section 4. Numerical experiments confirm the better conditioning of this ordering, which in turn implies a higher computational cost. We illustrate this low conditioning for equidistant nodes and also for the relevant case of Chebyshev nodes. Recall that Chebyshev nodes present almost optimal stability properties (see Section 1.3 of [5]). In Theorem 4.1, we show that $2^{n} n^{-1}+\frac{1}{2}$ is the corresponding upper bound for the conditioning of the Newton representation with equidistant nodes. The upper bound obtained in Theorem 4.1 is also used to improve some classical bounds of the Lebesgue constant for equidistant nodes.

\section{The conditioning of Newton formulae}

Let us recall the Lagrange interpolation problem by polynomials.

Lagrange interpolation problem Given a function $f \in C[a, b]$ and distinct nodes $x_{0}, \ldots, x_{n}$ in $[a, b]$, find a polynomial $p$ in $\mathbb{P}_{n}$, the space of polynomials of degree not greater than $n$, such that $p\left(x_{i}\right)=f\left(x_{i}\right)$, for $i=0, \ldots, n$.

The Lagrange interpolation problem has a unique solution $p$ given by the Lagrange formula

$$
p=\sum_{i=0}^{n} \lambda_{i} f l_{i}
$$

where

$$
\lambda_{i} f:=f\left(x_{i}\right), \quad i=0, \ldots, n,
$$

are the evaluation functionals and

$$
l_{i}(x):=\prod_{j \in\{0, \ldots, n\} \backslash\{i\}} \frac{x-x_{j}}{x_{i}-x_{j}}, \quad i=0, \ldots, n,
$$


are the Lagrange fundamental polynomials.

The Newton interpolation formula

$$
p=\sum_{i=0}^{n} d_{i} f \omega_{i}
$$

represents the interpolating polynomial in terms of the Newton monic polynomials

$$
\omega_{0}:=1, \quad \omega_{i}(x)=\left(x-x_{0}\right) \cdots\left(x-x_{i-1}\right), \quad i=1, \ldots, n+1,
$$

and the divided differences functionals

$$
d_{i} f:=\left[x_{0}, \ldots, x_{i}\right] f, \quad i=0, \ldots, n .
$$

Since the nodes are distinct, we have

$$
\left[x_{0}, \ldots, x_{i}\right] f=\sum_{k=0}^{i} \frac{f\left(x_{k}\right)}{\omega_{i+1}^{\prime}\left(x_{k}\right)},
$$

where

$$
\omega_{i+1}^{\prime}\left(x_{k}\right)=\prod_{j \in\{0, \ldots, i\} \backslash\{k\}}\left(x_{k}-x_{j}\right) .
$$

Let $L_{n}$ be the Lagrange interpolation operator

$$
L_{n}: f \in C[a, b] \rightarrow L_{n}[f] \in \mathbb{P}_{n},
$$

which associates to each function $f$ its Lagrange interpolation polynomial $L_{n}[f]$. For a given basis $v_{0}, \ldots, v_{n}$ of $\mathbb{P}_{n}$, the Lagrange interpolation operator can be represented in the form

$$
L_{n}[f]=\sum_{i=0}^{n} \beta_{i} f v_{i}
$$

where $\beta_{i}:=\sum_{j=0}^{n} c_{i j} \lambda_{j}, i=0, \ldots, n$, are suitable functionals belonging to the space generated by $\lambda_{0}, \ldots, \lambda_{n}$. The Lagrange representation $L_{n}[f]=\sum_{i=0}^{n} \lambda_{i} f l_{i}$ and the Newton representation $L_{n}[f]=\sum_{i=0}^{n} d_{i} f \omega_{i}$ are particular cases of (2.5).

For the purpose of analyzing the stability properties of a representation (2.5), we introduced in [1] a conditioning given by

$$
\operatorname{cond}(x ; \beta):=\sum_{i=0}^{n}\left\|\beta_{i}\right\|_{\infty}\left|v_{i}(x)\right|,
$$

where

$$
\left\|\beta_{i}\right\|_{\infty}:=\sup _{f \in C[a, b] \backslash\{0\}} \frac{\left|\beta_{i}[f]\right|}{\|f\|_{\infty}} .
$$


Letting $f$ in the previous definition bounded by -1 and 1 and equal to sign of $c_{i j}$ at $x_{i}$, we deduce that

$$
\left\|\beta_{i}\right\|_{\infty}=\sum_{j=0}^{n}\left|c_{i j}\right|
$$

(cf. Proposition 2 of [1]). The conditioning of the Lagrange representation coincides with the Lebesgue function $\lambda(x)$

$$
\lambda(x):=\sum_{i=0}^{n}\left|l_{i}(x)\right|=\sum_{i=0}^{n}|| \lambda_{i} \|_{\infty}\left|l_{i}(x)\right|=\operatorname{cond}(x ; \lambda)
$$

because $\left\|\lambda_{i}\right\|_{\infty}=1$, for $i=0, \ldots, n$. Observe that $\lambda(x)=L_{n}[f](x)$ for a function $f$ bounded by -1 and 1 and equal to sign of $l_{i}(x)$ at $x_{i}$. Using the triangular inequality

$$
\lambda(x)=\left|L_{n}[f](x)\right| \leq \sum_{i=0}^{n}\left|\beta_{i} f\right|\left|v_{i}(x)\right| \leq \sum_{i=0}^{n}\left\|\beta_{i}\right\|_{\infty}\left|v_{i}(x)\right|=\operatorname{cond}(x ; \beta)
$$

So, the conditioning of any other representation is greater than or equal to the conditioning of the Lagrange representation (cf. Theorem 4 of [1]), that is

$$
\operatorname{cond}(x ; \lambda) \leq \operatorname{cond}(x ; \beta) .
$$

Let us remark that the Newton representation depends on the order of the nodes. A different ordering of the nodes leads to different divided difference functionals $d_{0}, \ldots, d_{n}$ and different Newton monic polynomials $\omega_{0}, \ldots, \omega_{n}$. So, depending on the order of the nodes, the conditioning of the Newton formula

$$
\operatorname{cond}(x ; d)=\sum_{i=0}^{n}|| d_{i} \|_{\infty}\left|\omega_{i}(x)\right|
$$

may change.

The increasing or decreasing ordering of the nodes seems natural. However, it is well-known that the rounding error propagation grows quickly in a neighborhood of the last node. A successful alternative giving rise to good global results is provided by the Leja ordering (see [3], [4]).

Definition 2.1 (Leja order) A sequence of nodes $x_{0}, \ldots, x_{n}$ follows a Leja order if the nodes satisfy

$$
\prod_{j=0}^{k-1}\left|x_{k}-x_{j}\right| \geq \prod_{j=0}^{k-1}\left|x_{i}-x_{j}\right|, \quad i \geq k
$$


By (2.2), the Leja order chooses $x_{k}$ such that $\left|\omega_{k}\left(x_{k}\right)\right| \geq\left|\omega_{k}\left(x_{i}\right)\right|$, for $i \geq k$. In this paper we explore other orderings. One motivation to consider alternative orderings is providing a simplified criterion, comparing distances rather than products of distances. Moreover, tight bounds of $\operatorname{cond}(x ; d)$ for nodes following the Leja ordering are difficult to obtain.

The Lebesgue constant $\Lambda_{n}:=\max _{x \in[a, b]} \lambda(x)=\max _{x \in[a, b]} \operatorname{cond}(x ; \lambda)$ is the maximum value of the Lebesgue function. The particular case of equidistant nodes in increasing order in an interval $[a, b]$

$$
x_{i}=a+\frac{i}{h}, \quad h=\frac{b-a}{n}, \quad i=0, \ldots, n,
$$

leads to the following asymptotic formula by Schönhage [6] for the Lebesgue constant

$$
\Lambda_{n} \sim \frac{2^{n+1}}{e n \log (n+\gamma)},
$$

where $\gamma \approx 0.5772156649$ is the Euler-Mascheroni constant. In Corollary 12 of [1], the corresponding tight bound of the conditioning of the Newton representation

$$
\max _{x \in[a, b]} \operatorname{cond}(x ; d)=\operatorname{cond}\left(x_{n} ; d\right)=3^{n},
$$

was obtained. We observe that the asymptotic growth of the conditioning of the Newton representation is much greater than the conditioning of the Lagrange representation, in agreement with the optimality of the Lagrange representation shown in (2.7).

The following result will be used throughout this paper.

Lemma 2.1 Let $x_{0}, \ldots, x_{n}$ equidistant nodes satisfying (2.9) in $[a, b]$. Then

$$
\left\|\left[x_{\sigma(0)}, \ldots, x_{\sigma(n)}\right]\right\|_{\infty}=\frac{1}{n !}\left(\frac{2}{h}\right)^{n},
$$

for any permutation $\sigma:\{0, \ldots, n\} \rightarrow\{0, \ldots, n\}$.

Proof Formula (2.3) implies that the divided difference functional $\left[x_{\sigma(0)}, \ldots, x_{\sigma(n)}\right]$ coincides with $\left[x_{0}, \ldots, x_{n}\right]$ for any permutation $\sigma$. So, let us compute $\left\|\left[x_{0}, \ldots, x_{n}\right]\right\|_{\infty}$ for equidistant nodes. Using formulas (2.3) and (2.6), we have

$$
\left\|\left[x_{0}, \ldots, x_{n}\right]\right\|_{\infty}=\sum_{k=0}^{n} \frac{1}{\left|\omega_{n+1}^{\prime}\left(x_{k}\right)\right|} .
$$

Taking into account (2.4), we derive

$$
\begin{aligned}
& \left\|\left[x_{0}, \ldots, x_{n}\right]\right\|_{\infty}=\sum_{k=0}^{n} \frac{1}{\prod_{j \in\{0, \ldots, n\} \backslash\{k\}}\left|x_{k}-x_{j}\right|} \\
& =\frac{1}{h^{n}} \sum_{k=0}^{n} \frac{1}{\prod_{j \in\{0, \ldots, n\} \backslash\{k\}}|k-j|}=\frac{1}{n ! h^{n}} \sum_{k=0}^{n}\left(\begin{array}{l}
n \\
k
\end{array}\right)=\frac{1}{n !}\left(\frac{2}{h}\right)^{n} .
\end{aligned}
$$




\section{Central order}

Let us introduce an ordering of the nodes called central order.

Definition 3.1 (Central order) A sequence of nodes $x_{0}, \ldots, x_{n}$ follows a central order with respect to a center $c$ if the nodes satisfy

$$
\left|x_{0}-c\right| \leq\left|x_{1}-c\right| \leq \cdots \leq\left|x_{n}-c\right| \text {. }
$$

Central order may not be unique. Non-uniqueness can occur if there are nodes at the same distance of the center $c$.

If $x_{0}, \ldots, x_{n} \in[a, b]$, then the central order with respect to $a$ (respectively, $b$ ) is unique and coincides with the increasing (respectively, decreasing) order.

Remark 3.1 If the nodes are ordered following a central order, the convex hull of the sequence $x_{0}, \ldots, x_{k}$ is an interval not containing any node $x_{j}$ with $j>k$.

For the sake of simplicity, we consider the important case $c=0$ on symmetric intervals of the form $[-a, a]$. At the end of this section we will illustrate the influence of the choice of different centers.

In the following result, we bound a generalized binomial coefficient.

Proposition 3.1 Let $k$ be an integer and $\left(\begin{array}{l}x \\ k\end{array}\right):=x(x-1) \cdots(x-k+1) / k$ !. Then

$$
\left|\left(\begin{array}{l}
x \\
k
\end{array}\right)\right| \leq\left(\begin{array}{c}
m \\
k
\end{array}\right), \quad k-1-m \leq x \leq m,
$$

for any $m \geq k$.

Proof Let us use induction on $k$. For $k=0,1$, it is trivial. Let us assume that the inequality holds for $k-1$ and let us show it for $k$. If $x \in[0, k-1]$,

$$
\left|\left(\begin{array}{l}
x \\
k
\end{array}\right)\right|=\left|\frac{x-k+1}{k}\right|\left|\left(\begin{array}{c}
x \\
k-1
\end{array}\right)\right| \leq \frac{k-1}{k}\left(\begin{array}{c}
m-1 \\
k-1
\end{array}\right) \leq\left(\begin{array}{c}
m \\
k
\end{array}\right) .
$$

Let us observe that $\left(\begin{array}{l}x \\ k\end{array}\right)$ is an increasing function on $[k-1,+\infty)$ and then

$$
0 \leq\left(\begin{array}{l}
x \\
k
\end{array}\right) \leq\left(\begin{array}{c}
m \\
k
\end{array}\right), \quad x \in[k-1, m] .
$$

If $x \leq 0$, then $\left|\left(\begin{array}{l}x \\ k\end{array}\right)\right|=\left(\begin{array}{c}k-1-x \\ k\end{array}\right)$ is a decreasing function of $x$. So

$$
\left|\left(\begin{array}{l}
x \\
k
\end{array}\right)\right| \leq\left(\begin{array}{c}
m \\
k
\end{array}\right), \quad x \in[k-1-m, 0]
$$

We present an upper bound for $\operatorname{cond}(x ; d)$ at equidistant nodes following a central order. 
Theorem 3.1 Let $x_{0}, \ldots, x_{n} \in\{-a+2 a i / n, i=0, \ldots, n\}$ be a sequence following a central order with respect to $c=0$. Then, we have

$$
\operatorname{cond}(x ; d) \leq \frac{(1+\sqrt{2})^{n+1}+(1-\sqrt{2})^{n+1}}{2}
$$

Proof We denote by $h:=2 a / n$. By Remark 3.1, a central order always gives rise to subsets of equidistant nodes $\left\{x_{0}, \ldots, x_{k}\right\}$, for any $k \leq n$. So, by Lemma 2.1

$$
\left\|d_{k}\right\|_{\infty}=\frac{1}{k !}\left(\frac{2}{h}\right)^{k}, \quad k=0, \ldots, n .
$$

Recall that $\omega_{k}(x)=\prod_{i=0}^{k-1}\left(x-x_{i}\right), k=1, \ldots, n+1$ and $\omega_{0}(x)=1$. Let $n$ be even. If $k$ is odd, $k<n$, the first $k$ nodes are $j h, j=-(k-1) / 2, \ldots,(k-1) / 2$. In order to bound $\omega_{k}$, we apply Proposition 3.1

$$
\left|\omega_{k}(x)\right|=\prod_{j=-(k-1) / 2}^{(k-1) / 2}|x-j h|=h^{k} k !\left|\left(\begin{array}{c}
\frac{x}{h}+\frac{k-1}{2} \\
k
\end{array}\right)\right| \leq h^{k} k !\left(\begin{array}{c}
\frac{n+k-1}{2} \\
k
\end{array}\right),
$$

for $x \in[-a, a]$. If $k$ is even, using the previous bound, we derive

$$
\left|\omega_{k}(x)\right|=\left|\omega_{k-1}(x)\right|\left|x \pm \frac{k h}{2}\right| \leq h^{k} k !\left(\begin{array}{c}
\frac{n+k}{2} \\
k
\end{array}\right)
$$

for $x \in[-a, a]$.

Using (2.8) and the above formulae, we have

$$
\operatorname{cond}(x ; d)=\sum_{k=0}^{n}\left\|d_{k}\right\|_{\infty}\left|\omega_{k}(x)\right| \leq \sum_{k=0}^{n}\left(\begin{array}{c}
n / 2+\left\lfloor\frac{k}{2}\right\rfloor \\
k
\end{array}\right) 2^{k}=s_{n}+2 t_{n},
$$

where

$$
s_{n}:=\sum_{\substack{k=0 \\
k \text { even }}}^{n}\left(\begin{array}{c}
n / 2+\frac{k}{2} \\
k
\end{array}\right) 2^{k}=\sum_{k=0}^{n / 2}\left(\begin{array}{c}
n / 2+k \\
2 k
\end{array}\right) 2^{2 k}
$$

and

$$
t_{n}:=\frac{1}{2} \sum_{\substack{k=1 \\
k \text { odd }}}^{n-1}\left(\begin{array}{c}
n / 2+\frac{k-1}{2} \\
k
\end{array}\right) 2^{k}=\sum_{k=0}^{n / 2-1}\left(\begin{array}{c}
n / 2+k \\
2 k+1
\end{array}\right) 2^{2 k} .
$$

Then, we have

$$
\begin{aligned}
& s_{n}+t_{n}=\sum_{k=0}^{n / 2}\left(\begin{array}{c}
n / 2+k \\
2 k
\end{array}\right) 2^{2 k}+\sum_{k=0}^{n / 2-1}\left(\begin{array}{c}
n / 2+k \\
2 k+1
\end{array}\right) 2^{2 k}= \\
& \sum_{k=0}^{n / 2}\left(\begin{array}{c}
n / 2+k+1 \\
2 k+1
\end{array}\right) 2^{2 k}=t_{n+2},
\end{aligned}
$$


and

$$
\begin{aligned}
& s_{n}+4 t_{n+2}=\sum_{k=0}^{n / 2}\left(\begin{array}{c}
n / 2+k \\
2 k
\end{array}\right) 2^{2 k}+4 \sum_{k=0}^{n / 2}\left(\begin{array}{c}
n / 2+k+1 \\
2 k+1
\end{array}\right) 2^{2 k}= \\
& \sum_{k=0}^{n / 2}\left(\begin{array}{c}
n / 2+k \\
2 k
\end{array}\right) 2^{2 k}+\sum_{k=1}^{n / 2+1}\left(\begin{array}{c}
n / 2+k \\
2 k-1
\end{array}\right) 2^{2 k}=\sum_{k=0}^{n / 2+1}\left(\begin{array}{c}
n / 2+k+1 \\
2 k
\end{array}\right) 2^{2 k}=s_{n+2} .
\end{aligned}
$$

Therefore, $s_{n+2}=5 s_{n}+4 t_{n}$. Thus, we have the following difference equation

$$
\left(\begin{array}{l}
s_{n+2} \\
t_{n+2}
\end{array}\right)=\left(\begin{array}{ll}
5 & 4 \\
1 & 1
\end{array}\right)\left(\begin{array}{l}
s_{n} \\
t_{n}
\end{array}\right), \quad\left(\begin{array}{l}
s_{0} \\
t_{0}
\end{array}\right)=\left(\begin{array}{l}
1 \\
0
\end{array}\right),
$$

whose solution is

$$
\left(\begin{array}{c}
s_{n} \\
t_{n}
\end{array}\right)=\frac{1}{4 \sqrt{2}}\left[(1+\sqrt{2})^{n}\left(\begin{array}{c}
2(1+\sqrt{2}) \\
1
\end{array}\right)-(1-\sqrt{2})^{n}\left(\begin{array}{c}
2(1-\sqrt{2}) \\
1
\end{array}\right)\right] .
$$

Consequently, $\operatorname{cond}(x ; d)$ for even $n$ can be bounded with the following value

$$
\begin{aligned}
s_{n}+2 t_{n} & =\frac{(1+\sqrt{2})^{n+1}}{2 \sqrt{2}}-\frac{(1-\sqrt{2})^{n+1}}{2 \sqrt{2}}+\frac{(1+\sqrt{2})^{n}}{2 \sqrt{2}}-\frac{(1-\sqrt{2})^{n}}{2 \sqrt{2}} \\
& =\frac{1}{2}(1+\sqrt{2})^{n+1}+\frac{1}{2}(1-\sqrt{2})^{n+1} .
\end{aligned}
$$

For $n$ odd, we proceed in the same way. If $k$ is even, $k<n$, the first $k$ nodes are $(j-1 / 2) h, j=-k / 2+1, \ldots, k / 2$. Using Proposition 3.1, we have

$$
\left|\omega_{k}(x)\right|=\prod_{j=-k / 2+1}^{k / 2}\left|x-\left(j-\frac{1}{2}\right) h\right|=h^{k} k !\left|\left(\begin{array}{c}
\frac{x}{h}+\frac{k-1}{2} \\
k
\end{array}\right)\right| \leq h^{k} k !\left(\begin{array}{c}
\frac{n+k-1}{2} \\
k
\end{array}\right),
$$

for all $x \in[-a, a]$. For odd $k$

$$
\left|\omega_{k}(x)\right|=\left|\omega_{k-1}(x)\right|\left|x \pm \frac{k h}{2}\right| \leq h^{k} k !\left(\begin{array}{c}
\frac{n+k}{2} \\
k
\end{array}\right)
$$

for all $x \in[-a, a]$.

Using (2.8) and the above formulae, we have

$$
\begin{aligned}
\operatorname{cond}(x ; d) & =\sum_{k=0}^{n}\left\|d_{k}\right\|_{\infty}\left|\omega_{k}(x)\right| \leq \sum_{k=0}^{n}\left(\begin{array}{c}
\frac{n-1}{2}+\left\lfloor\frac{k+1}{2}\right\rfloor \\
k
\end{array}\right) 2^{k} \\
& =\sum_{k=0}^{(n-1) / 2}\left(\begin{array}{c}
(n-1) / 2+k \\
2 k
\end{array}\right) 2^{2 k}+\sum_{k=0}^{(n-1) / 2}\left(\begin{array}{c}
(n+1) / 2+k \\
2 k+1
\end{array}\right) 2^{2 k+1} .
\end{aligned}
$$

Therefore, the conditioning for odd $n$ can be bounded as

$$
\operatorname{cond}(x ; d) \leq s_{n-1}+2 t_{n+1} .
$$


Using (3.1), we have

$$
\begin{aligned}
s_{n-1}+2 t_{n+1} & =\frac{(1+\sqrt{2})^{n}}{2 \sqrt{2}}-\frac{(1-\sqrt{2})^{n}}{2 \sqrt{2}}+\frac{(1+\sqrt{2})^{n+1}}{2 \sqrt{2}}-\frac{(1-\sqrt{2})^{n+1}}{2 \sqrt{2}} \\
& =\frac{1}{2}(1+\sqrt{2})^{n+1}+\frac{1}{2}(1-\sqrt{2})^{n+1} \cdot
\end{aligned}
$$

Remark 3.2 Let us recall that in a central order we can choose between two nodes at the same distance to the center. If we first choose the least node between both nodes, then the bound of Theorem 3.1 is attained at $x=a$. Analogously, the bound is attained at $x=-a$ if we choose the greatest node.

Taking into account the equality (2.11), the previous theorem shows that $C_{n}:=\max _{x \in[a, b]} \operatorname{cond}(x ; d)$ is lower for equidistant nodes following any central order than in increasing order. In both cases $C_{n}$ presents an exponential growth which can be measured by $r:=\limsup _{n \rightarrow \infty} C_{n}^{1 / n}$. For increasing order, $r=3$, and for a central order, $r \leq 1+\sqrt{2}$. By (2.7), the Lagrange representation has optimal conditioning and, by (2.10), the corresponding ratio is $r=\lim _{n \rightarrow \infty} \Lambda_{n}^{1 / n}=2$.

We have tested numerically the bound in Theorem 3.1 in the case of equidistant nodes following a central order with respect to the center $c=0$ in the interval $[-a, a]$. Inspired by Remark 3.2, we propose the following criterion in order to achieve lower values of $\max _{x \in[-a, a]} \operatorname{cond}(x ; d)$. If we have two nodes with the same distance to zero, we choose as next node the one which is on the same side of the origin as the previous node. We distinguish two cases. For even $n, i=0, \ldots, n$, we take

$$
x_{i}= \begin{cases}-(-1)^{i / 2} \frac{i h}{2}, & \text { if } i \text { even, } \\ -(-1)^{(i-1) / 2} \frac{(i+1) h}{2}, & \text { if } i \text { odd, }\end{cases}
$$

and for odd $n, i=0, \ldots, n$,

$$
x_{i}= \begin{cases}-(-1)^{i / 2} \frac{(i+1) h}{2}, & \text { if } i \text { even, } \\ (-1)^{(i-1) / 2 \frac{i h}{2},} & \text { if } i \text { odd. }\end{cases}
$$

Figure 3.1 shows $\log _{3}\left(\lambda_{L}(x)\right)$ at equidistant nodes and $\log _{3}(\operatorname{cond}(x ; d))$ at equidistant nodes in $[-1,1]$ for $n=10$ with different orderings: the increasing order, a Leja order and the central order given by (3.2). The increasing order gives the highest values for $\operatorname{cond}(x ; d)$ close to the right end of the interval. By Theorem 3.1, the upper bound for the central order is lower than the upper bound for the increasing order, as shown in the figure. Besides, the central order has the best behaviour in a neighborhood of the center. Finally, a Leja ordering has a better global behaviour.

Figure 3.2 compares $\log _{3}\left(\lambda_{L}(x)\right)$ and $\log _{3}(\operatorname{cond}(x ; d))$ at equidistant nodes following a central order on $[-1,1]$ for $n=19$ with centers $c=2 / 3$ and 


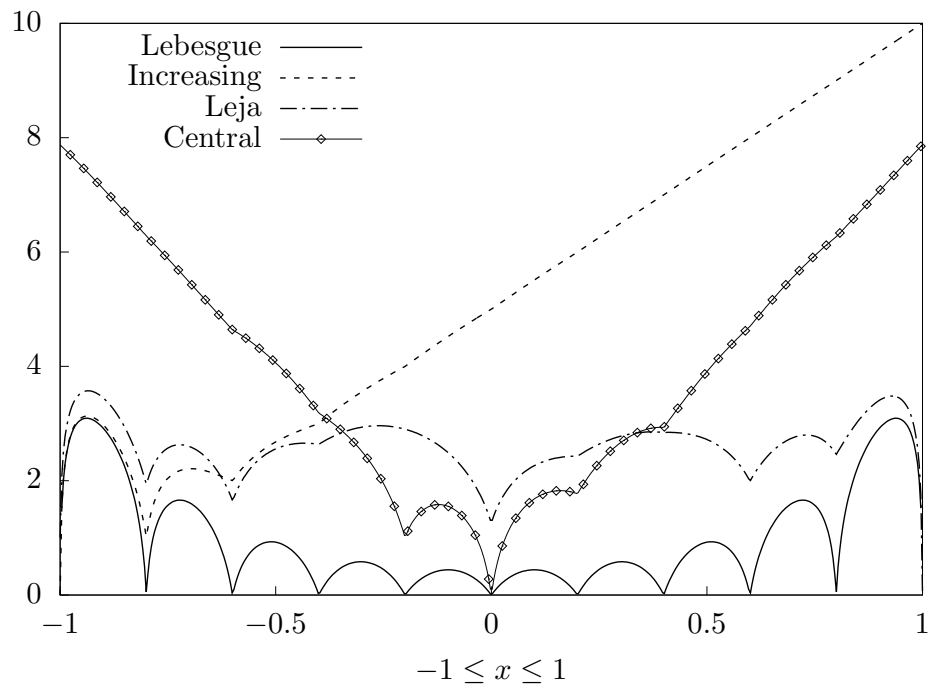

Fig. 3.1 $\log _{3}\left(\lambda_{L}(x)\right), \log _{3}(\operatorname{cond}(x ; d))$ for equidistant nodes in increasing order, in a Leja order and in a central order respect to $c=0$ in $[-1,1]$ for $n=10$.

$c=-2 / 3$, respectively. We observe that, in both cases, in a neighborhood of the center $\operatorname{cond}(x ; d)$ is similar to the Lebesgue constant and it grows quickly when we move away from $c$.

\section{Central order with respect to the evaluation point}

In the central order, the center $c$ is fixed. Now, we propose to order the nodes according to the distance to the evaluation point $x$, that is,

$$
\left|x-x_{0}\right| \leq\left|x-x_{1}\right| \leq \cdots \leq\left|x-x_{n}\right| .
$$

Remark 4.1 We observe that if $x_{0}, \ldots, x_{n}$ are nodes following central order with respect to the evaluation point (4.1), then we have

$\left|\omega_{k}(x)\right|=\left|\left(x-x_{0}\right) \cdots\left(x-x_{k-1}\right)\right| \leq\left|\left(x-x_{\sigma(0)}\right) \cdots\left(x-x_{\sigma(k-1)}\right)\right|, \quad k=0, \ldots, n$, for any permutation $\sigma:\{0, \ldots, n\} \rightarrow\{0, \ldots, n\}$. Indeed, by ordering each factor $\left|x-x_{\sigma(i)}\right|, i=0, \ldots, k-1$, from the lowest to the highest, we see that each of these factors is greater than or equal to the corresponding one $\left|x-x_{i}\right|$, $i=0, \ldots, k-1$.

Remark 4.1 suggests that this order is well conditioned. In fact, the conditioning of the Newton representation with nodes following central order with respect to the evaluation point is closer to the Lebesgue function than with other orderings, such as Leja ordering. Figure 4.1 shows that the Leja order is 

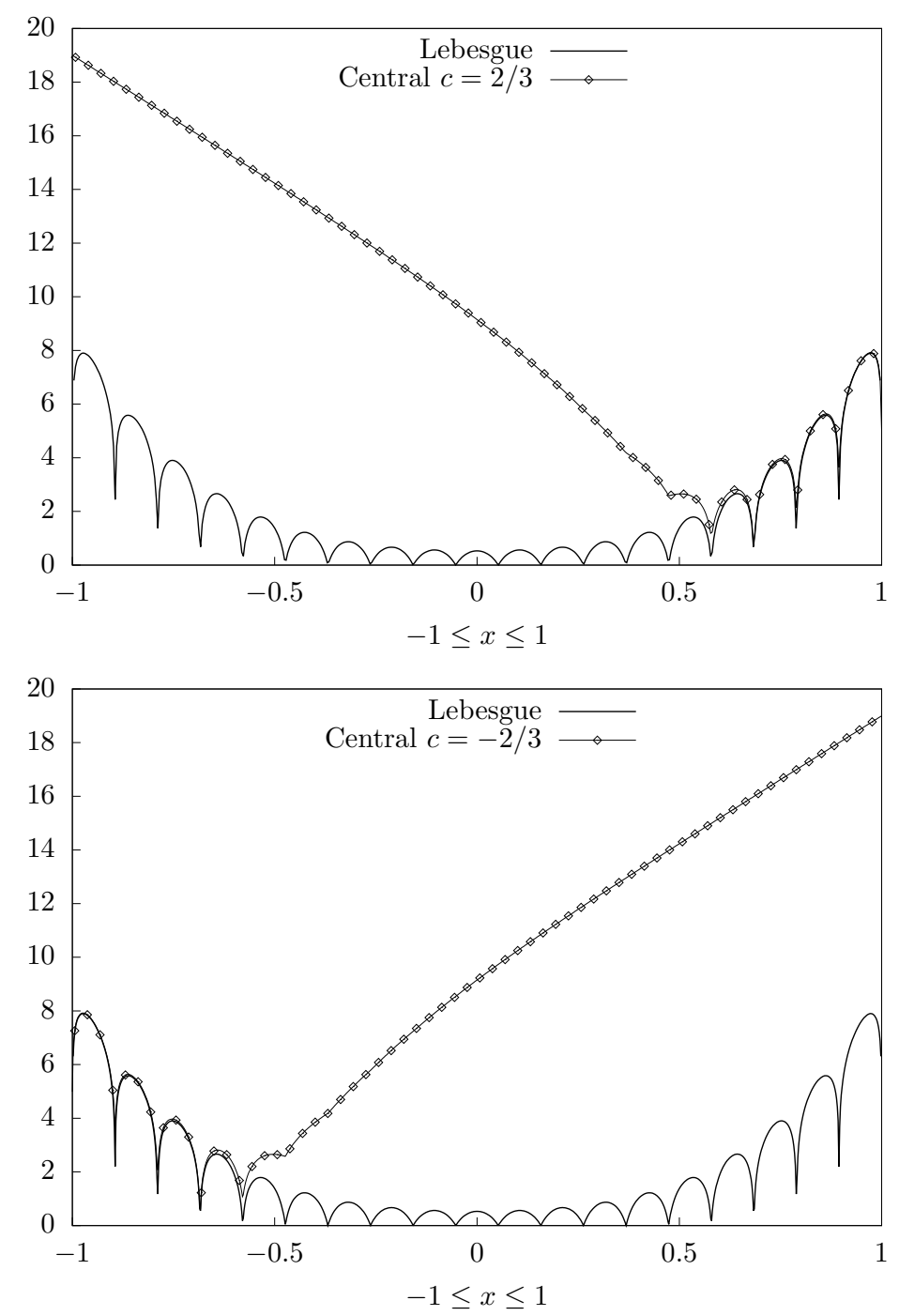

Fig. 3.2 $\log _{3}\left(\lambda_{L}(x)\right)$ and $\log _{3}(\operatorname{cond}(x ; d))$ for equidistant nodes in central order with respect to $c=2 / 3$ (up) and with respect to $c=-2 / 3$ (down) for $n=19$.

worse conditioned than the central order with respect to the evaluation point for Chebyshev nodes of degree $n=10$.

Let us show an auxiliary inequality to bound $\operatorname{cond}(x ; d)$.

Lemma 4.1 Let $1 \leq i \leq n-1$. Then

$$
\sum_{k=i+1}^{n} \frac{2^{k}}{k} \leq \frac{2^{n+2}}{n}-3 \frac{2^{i+1}}{i+1}
$$




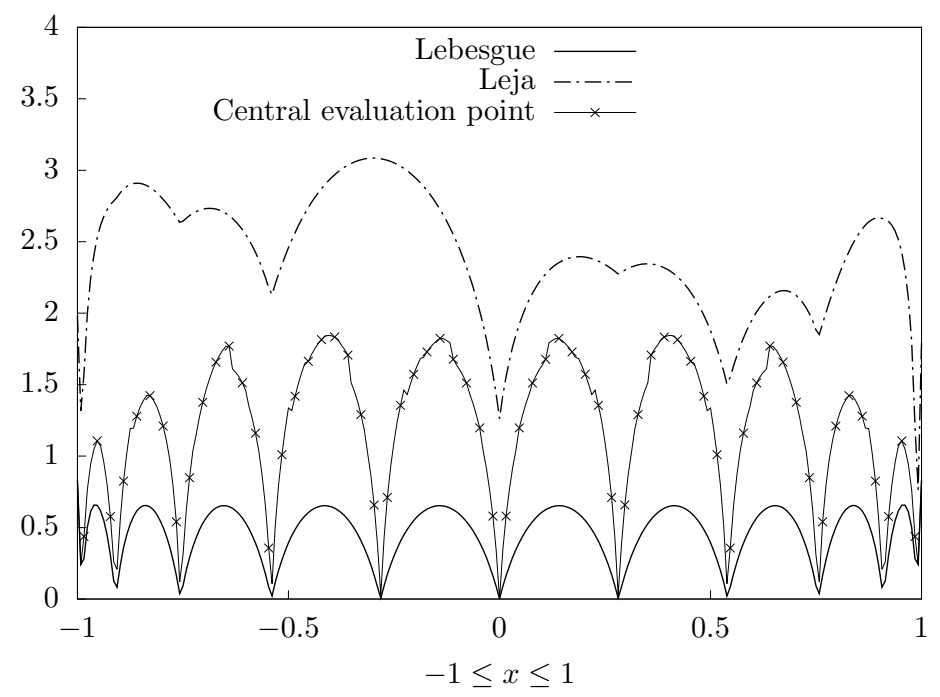

Fig. 4.1 $\log _{3}\left(\lambda_{L}(x)\right)$ and $\log _{3}(\operatorname{cond}(x ; d))$ for Chebyshev nodes with Leja order and central order with respect to the evaluation point for $n=10$.

Proof

$$
\begin{aligned}
\sum_{k=i+1}^{n} \frac{2^{k}}{k} & =2 \sum_{k=i+1}^{n} \frac{2^{k+1}}{k}-3 \sum_{k=i}^{n-1} \frac{2^{k+1}}{k+1} \\
& =\frac{2^{n+2}}{n}-3 \frac{2^{i+1}}{i+1}+\sum_{k=i+1}^{n-1}\left(\frac{2}{k}-\frac{3}{k+1}\right) 2^{k+1}
\end{aligned}
$$

For $k \geq 2, \frac{2}{k}-\frac{3}{k+1} \leq 0$ and then we obtain the result.

Let us observe that if we arrange the nodes in increasing order, we can compute a table of divided differences containing all divided differences corresponding to consecutive nodes. By Remark 3.1, the divided differences in this table can be used to compute any Newton formula for nodes following any central order and also for a central order with respect to the evaluation point. If we change the evaluation point, we might change some terms in the Newton formula (2.1). However, divided differences need not be recomputed and can be searched in the table.

A disadvantage of central ordering with respect to the evaluation point is the higher computation cost because the nodes might be reordered for each evaluation point. However, for certain nodes sets (for example, equidistant nodes) strategies can be designed to reduce this cost.

Remark 4.2 The number of the central orderings with respect to the evaluation point is bounded above by $n(n+1) / 2+1$. Assume that $x_{0} \leq x_{1} \leq \cdots \leq x_{n}$, that is, the nodes follow an increasing order. If $x<\left(x_{0}+x_{1}\right) / 2$ then the central order 
with respect to $x$ coincides with the increasing order. Let $x_{i j}=\left(x_{i}+x_{j}\right) / 2$ be the bisection point of the nodes $x_{i}$ and $x_{j}$. If the evaluation point moves from left to right, it is convenient to sort all the bisection points. Each time that $x$ surpasses a bisection point $x_{i j}$ it is necessary to update the order by exchanging $x_{i}$ and $x_{j}$. If several bisection points coincide then we have to exchange the corresponding pairs of nodes. At the end, if $x$ is greater than the last bisection point, then the central order coincides with the decreasing order. In the particular case the nodes are equidistant, there are only $2 n$ orderings and $2 n-1$ bisection points: the mid points of each pair of consecutive nodes and $x_{1}, \ldots, x_{n-1}$.

In the following result we provide an upper bound for $\operatorname{cond}(x ; d)$ when equidistant nodes follow the central order with respect to the evaluation point.

Theorem 4.1 Let $x_{0}, \ldots, x_{n}$ be equidistant nodes satisfying (4.1) with respect to $x$ in $[a, b]$. Then, we have

$$
\operatorname{cond}(x ; d) \leq \frac{2^{n}}{n}+\frac{1}{2}
$$

Proof By Remark 3.1, the ordering (4.1) always gives rise to subsets $\left\{x_{0}, \ldots, x_{k}\right\}$ consisting in equidistant nodes, for $k \leq n$. So, by Lemma 2.1

$$
\left\|d_{k}\right\|_{\infty}=\frac{1}{k !}\left(\frac{2}{h}\right)^{k}, \quad k=0, \ldots, n .
$$

From (4.1), we deduce that $x$ is between $x_{0}$ and $x_{1}$, with $\left|x_{1}-x_{0}\right|=h$. We have

$$
\left|x-x_{0}\right| \leq \frac{h}{2}, \quad\left|x-x_{1}\right| \leq h
$$

and

$$
\left|\omega_{2}(x)\right|=\left|x-x_{0}\right|\left|x-x_{1}\right| \leq \frac{h^{2}}{4} .
$$

Besides, $\min _{j=0, \ldots, i}\left|x_{i+1}-x_{j}\right|=h$. Let $j \leq i$ such that $\left|x_{i+1}-x_{j}\right|=h$. Since $x_{0}, \ldots, x_{n}$ follow a central order, we have

$$
\left|x-x_{i+1}\right| \leq\left|x-x_{j}\right|+\left|x_{i+1}-x_{j}\right| \leq\left|x-x_{i}\right|+h .
$$

By induction on $i$, we deduce that

$$
\left|x-x_{i}\right| \leq i h, \quad i \geq 1 .
$$

Thus,

$$
\begin{aligned}
\left|\omega_{k}(x)\right| & =\left|\left(x-x_{0}\right)\left(x-x_{1}\right) \cdots\left(x-x_{k-1}\right)\right| \leq \frac{h^{2}}{4} \cdot 2 h \cdot 3 h \cdots(k-1) h \\
& =\frac{h^{k}}{4}(k-1) !, \quad k=2, \ldots, n .
\end{aligned}
$$


By (2.8), the above formulae and Lemma 4.1

$$
\begin{aligned}
\operatorname{cond}(x ; d) & =\sum_{k=0}^{n}|| d_{k} \|_{\infty}\left|\omega_{k}(x)\right|=2+\sum_{k=2}^{n}|| d_{k}||_{\infty}\left|\omega_{k}(x)\right| \\
& \leq 2+\sum_{k=2}^{n}\left(\frac{2}{h}\right)^{k} \frac{1}{k !} \frac{h^{k}}{4}(k-1) ! \\
& =2+\frac{1}{4} \sum_{k=2}^{n} \frac{2^{k}}{k} \leq 2+\frac{1}{4}\left(\frac{2^{n+2}}{n}-3 \frac{2^{2}}{2}\right)=\frac{2^{n}}{n}+\frac{1}{2} .
\end{aligned}
$$

Applying Theorem 4.1, we obtain in a simple way a bound of the Lebesgue constant close to formula (2.10).

Corollary 4.1 The Lebesgue function at equidistant nodes in $[a, b]$ satisfies

$$
\lambda_{L}(x) \leq \frac{2^{n}}{n}+\frac{1}{2}, \quad x \in[a, b] .
$$

Proof For each $x \in[a, b]$, we order the nodes according to (4.1). Using formula (2.7) and Theorem 4.1, we have

$$
\lambda_{L}(x) \leq \operatorname{cond}(x ; d) \leq \frac{2^{n}}{n}+\frac{1}{2} .
$$

Let us recall the bound of the Lebesgue constant

$$
\Lambda_{n} \leq 2^{n},
$$

based on a private communication by Jia Rong-Qing to Carl de Boor (see 1.3.22 of [5]). In Theorem 2 of [7], Trefethen and Weideman deduced the following improved upper bound

$$
\Lambda_{n} \leq \frac{2^{n+3}}{n} .
$$

Note that Corollary 4.1 improves the previous bound because

$$
\frac{2^{n}}{n}+\frac{1}{2} \leq \frac{2^{n+3}}{n}, \quad n \geq 1 .
$$

Figure 4.2 shows that $\operatorname{cond}(x ; d)$ at equidistant nodes following the central order with respect to $x$ is close to the Lebesgue function in a neighborhood of each node, while the difference is greater in a neighborhood of the center of each subinterval. Besides, central order with respect to the evaluation point presents a better behaviour than a Leja ordering.

Acknowledgements This work has been partially supported by the Spanish Research Grant MTM2015-65433-P (MINECO/FEDER), BES-2013-065398B (MINECO), by Gobierno the Aragón and Fondo Social Europeo. 


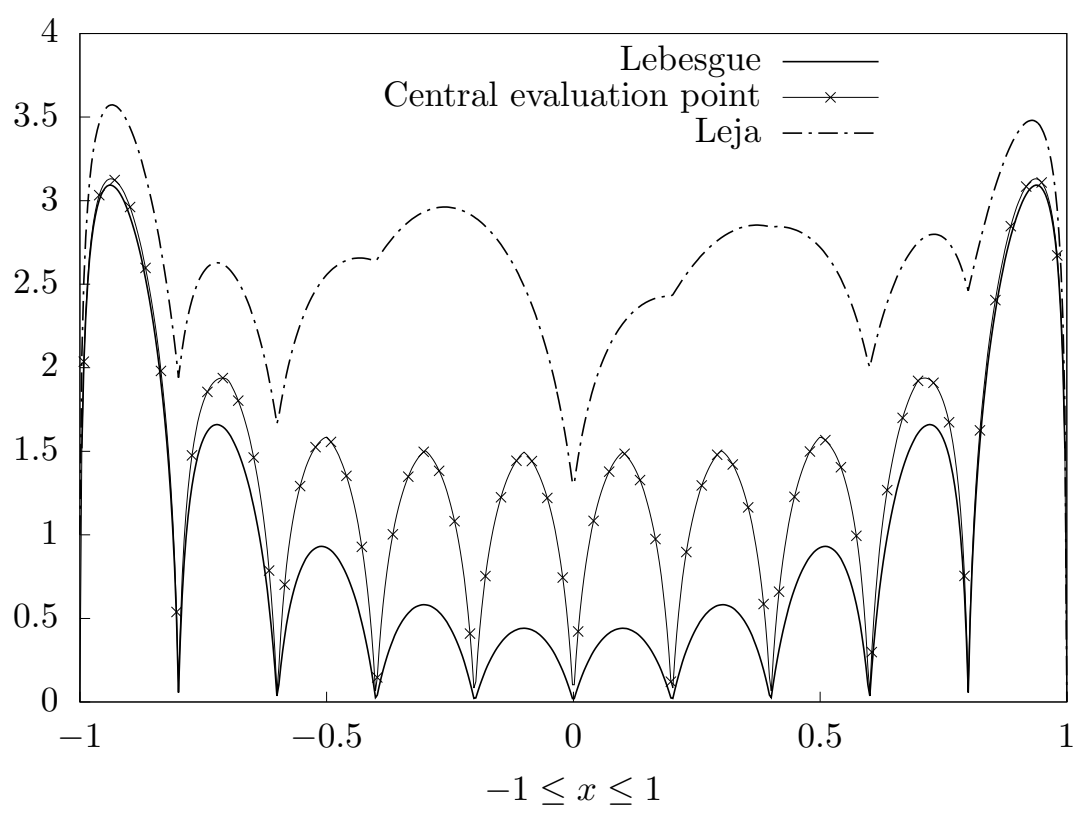

Fig. 4.2 $\log _{3}\left(\lambda_{L}(x)\right)$ and $\log _{3}(\operatorname{cond}(x ; d))$ for equidistant nodes in Leja order and central order with respect to the evaluation point for $n=10$.

\section{References}

1. J. M. Carnicer, Y. Khiar, J. M. Peña, Optimal stability of the Lagrange formula and conditioning of the Newton formula, to appear in J. Approx. Theory, DOI: 10.1016/j.jat.2017.07.005.

2. R. M. Corless, S. M. Watt, Bernstein bases are optimal, but, sometimes, Lagrange bases are better, in Proc. SYNASC (Symbolic and Numeric Algorithms for Scientific Computing), pp. 141-152, Timisoara (2004).

3. F. Leja, Sur certaines suites liées aux ensembles plans et leur application à la représentation conforme, Ann. Polon. Math., 4, 8-13 (1957).

4. L. Reichel, Newton interpolation at Leja points, BIT, 30, 332-346 (1990).

5. T. J. Rivlin, Chebyshev polynomials. From approximation theory to algebra and number theory. Pure and Applied Mathematics, John Wiley \& Sons, Inc., New York (1990).

6. A. Schönhage, Fehlerfortpflanzung bei Interpolation, Numer. Math, 3, 62-71 (1961).

7. L. N. Trefethen, J. A. C. Weideman, Two results on polynomial interpolation in equally spaced points, J. Approx. Theory, 65, 247-260 (1991). 